PROCEEDINGS OF THE

AMERICAN MATHEMATICAL SOCIETY

Volume 126, Number 5, May 1998, Pages 1461-1466

S 0002-9939(98)03846-5

\title{
*-REPRESENTATIONS ON BANACH *-ALGEBRAS
}

\author{
A. K. GAUR
}

(Communicated by Palle E. T. Jorgensen)

\begin{abstract}
We study notions of $g$-bounded linear functionals and representable functionals on Banach *-algebras. An equivalence between these two is established for general Banach *-algebras. In particular, we characterize $g$ bounded linear functionals on Banach *-algebras with approximate identity and isometric involution. In addition, we prove a result on representation of $g$-bounded positive linear functionals in terms of cyclic vectors for the corresponding *-representation.
\end{abstract}

\section{INTRODUCTION}

Let $A$ be a complex Banach *-algebra. We assume neither the existence of an identity nor that the involution is continuous. We write $S(A)=\{a$ in $A$ such that $\left.a^{*}=a\right\}$ for the set of all self-adjoint elements of $A$. A *-ideal of $A$ is an ideal $J$ of $A$ where $a$ in $J$ implies $a^{*}$ in $J$.

A $B^{*}$-semi-norm on $A$ is a function $\eta: A \rightarrow \mathbb{R}$ such that for all $a, b$ in $A$ and $\alpha$ in $\mathbb{C}$,

(1) $\eta(a+b) \leq \eta(a)+\eta(b)$

(2) $\eta(\alpha a)=|\alpha| \eta(a)$

(3) $\eta(a b) \leq \eta(a) \cdot \eta(b)$,

(4) $\eta\left(a^{*} a\right)=(\eta(a))^{2}$.

$P(A)$ denotes the set of all $B^{*}$-semi-norms on $A$. For more on $B^{*}$-semi-norms, see $[2,3]$. Suppose $g(a)=\sup \{\eta(a): \eta$ in $P(A)\}$. Then $g$ defines a $B^{*}$-semi-norm on $A$; in fact, $g$ is the greatest $B^{*}$-semi-norm on $A$ in the pointwise ordering.

A *-representation of $A$ is a mapping $\pi: A \rightarrow B(H)$, where $B(H)$ denotes the algebra of all bounded linear operators on a Hilbert space $H$, such that for all $a, b$ in $A$ and $\alpha$ in $\mathbb{C}$

(1) $\pi(a+b)=\pi(a)+\pi(b)$,

(2) $\pi(\alpha a)=\alpha \pi(a)$

(3) $\pi(a b)=\pi(a) \cdot \pi(b)$,

(4) $\pi\left(a^{*}\right)=(\pi(a))^{*}$.

Received by the editors October 16, 1995 and, in revised form, February 14, 1996, August 19, 1996, September 10, 1996, and October 25, 1996; the contents of this paper were presented at the AMS San Francisco Meeting on January 6, 1995.

1991 Mathematics Subject Classification. Primary 46K15, 46H15.

Key words and phrases. Banach *-algebra, $g$-bounded linear functionals, representable functional, *-representation.

This research is supported by the Presidential Scholarship Award, 1995-96.

(C)1998 American Mathematical Society 
Note that every ${ }^{*}$-representation of $A$ is uniformly continuous, since each $B^{*}$-seminorm on $A$ is continuous and the mapping $a \rightarrow|\pi(a)|$ is a $B^{*}$-semi-norm on $A$.

A given positive linear functional $f$ on $A$ is representable if there exists a *representation $\pi$ of $A$ on $H$ and a vector $x$ in $H$ such that $f$ is the positive linear functional represented by $(\pi, x)$; that is, $f(a)=(\pi(a) x, x)$ for all $a \in A$.

\section{2. $g$-BOUNDED FUNCTIONALS}

Definition 2.1. A linear functional $f$ on $A$ is $g$-bounded if there exists a constant $M>0$ (depending only on $f$ ) such that for all $a$ in $A,|f(a)| \leq M g(a)$.

Since each $B^{*}$-semi-norm on $A$ is continuous, it follows that any $g$-bounded linear functional is continuous with respect to the original norm on $A$ and hence the set of $g$-bounded linear functionals is a subspace of $A^{*}$, the dual space of $A$. The norm of any $g$-bounded functional $f$ is defined as follows:

$$
|f|_{g}=\sup \{|f(a)|: g(a) \leq 1\} .
$$

The set $D(g)$ consists of all $g$-bounded positive linear functionals $f$ on $A$ with $|f|_{g} \leq 1$. A positive $g$-bounded linear functional $f$ on $A$ will be called a state of $A$ if $|f|_{g}=1$.

Lemma 2.1. Let $A$ be a unital algebra and $f$ be a positive g-bounded linear functional on $A$. Then $f$ is a state of $A$.

Proof. Since $f(1) \leq 1$, it follows that $|f|_{g} \geq f(1)$, where 1 is the identity element in the algebra $A$. But for all $a$ in $A$,

$$
|f(a)| \leq|f|_{g} g(a) .
$$

Hence for all $a$ in $A$,

$$
|f(a)|^{2} \leq f(1)|f|_{g} g\left(a^{*} a\right)=f(1)|f|_{g} g(a)^{2} .
$$

Thus $|f|_{g}^{2} \leq f(1)|f|_{g}$ and consequently $f$ is a state of $A$.

Proposition 2.1. Let $u$ and $v$ be positive g-bounded linear functionals on A. Then for all a in $A$

(i) $|u(a)|^{2} \leq|u|_{g} u\left(a^{*} a\right)$,

(ii) $|u+v|_{g}=|u|_{g}+|v|_{g}$,

(iii) $|u|_{g}=\sup \left\{u\left(a^{*} a\right): g(a) \leq 1\right\}$,

(iv) $u$ and $v$ are hermitian functionals.

Proof. Suppose that $A$ has no unit element. If $J_{g}=\{a$ in $A: g(a)=0\}$, then $J_{g}$ is a closed two-sided *-ideal of $A$. In that case $A / J_{g}$ becomes a quotient *-algebra. Let $a \rightarrow \lambda_{a}$ denote the canonical mapping of $A$ onto $A / J_{g}$. We define a $B^{*}$-norm $\bar{g}$ on $A / J_{g}$ as follows: For all $a$ in $A / J_{g}, \bar{g}\left(\lambda_{a}\right)=g(a)$. The completion $A_{g}$ of $A / J_{g}$ with respect to this norm is a $B^{*}$-algebra. On $A / J_{g}$, define $\bar{u}\left(\lambda_{a}\right)=u(a)$, $\bar{v}\left(\lambda_{a}\right)=v(a)$, for all $\lambda_{a}$ in $A / J_{g}$. Then $\bar{u}$ and $\bar{v}$ are well defined positive $\bar{g}$-bounded linear functionals on $A / J_{g}$. Furthermore, $|\bar{u}|_{\bar{g}}=|u|_{g}$ and $|\bar{v}|_{\bar{g}}=|v|_{g}$. Hence $\bar{u}$ and $\bar{v}$ have a unique norm preserving extension to the $B^{*}$-algebra $A_{g}$. Denote these extensions by $U$ and $V$, respectively.

Thus $U$ and $V$ are positive linear functionals on $A_{g}$, hence (i), (ii), and (iv) follow from 2.1.5 and 2.1.6 in [1]. To prove (iii) we proceed as follows. Let $\sup \left\{u\left(a^{*} a\right)\right.$ : $g(a) \leq 1\}=\alpha$. Then $\alpha \leq|u|_{g}$, since if $g(a) \leq 1, u\left(a^{*} a\right) \leq|u|_{g} g\left(a^{*} a\right) \leq|u|_{g}$. By the definition of $|u|_{g}$ there exists a sequence $\left\{a_{k}\right\}$ of elements of $A$ with $g\left(a_{k}\right) \leq 1$ and $|u|_{g}=\lim _{k \rightarrow \infty}\left|u\left(a_{k}\right)\right|$. 
It follows from (i) that

$$
\left|u\left(a_{k}\right)\right|^{2} \leq|u|_{g} u\left(a_{k}^{*} a_{k}\right) \leq|u|_{g}^{2} g\left(a_{k}\right)^{2} \leq|u|_{g}^{2} .
$$

Hence $\lim _{k \rightarrow \infty} u\left(a_{k}^{*} a_{k}\right)=|u|_{g}$. The case where $A$ is unital follows from Lemma 2.1 above.

Remark 2.1. Proposition 2.1 is also true for any $B^{*}$-semi-norm. The following theorem gives a necessary and a sufficient condition for a positive functional on an algebra to be $g$-bounded.

Theorem 2.1. A positive linear functional $f$ on $A$ is $g$-bounded if and only if there exists a positive constant $M$, which depends only on $f$, such that for all a in $A$, $|f(a)|^{2} \leq M f\left(a^{*} a\right)$.

Moreover if $f$ is $g$-bounded, then

$$
|f|_{g}=\sup _{a \in A}\left\{\frac{|f(a)|^{2}}{f\left(a^{*} a\right)}\right\} .
$$

Proof. Let $f$ be a $g$-bounded positive functional. Then by Proposition 2.1(i) it follows that for all $a$ in $A,|f(a)|^{2} \leq M f\left(a^{*} a\right)$.

Suppose conversely that for all $a$ in $A$, and for every positive linear functional $f$ on $A$, there exists a positive constant $M$ such that $|f(a)|^{2} \leq M f\left(a^{*} a\right)$. Then for all $x$ and $a$ in $A$

$$
\beta_{f}=\sup _{f\left(x^{*} x\right) \leq 1}\left\{\sqrt{f\left(x^{*} a^{*} a x\right)}\right\} \geq \sup _{f\left(x^{*} x\right) \leq 1}\left\{\frac{|f(a x)|}{\sqrt{M}}\right\} .
$$

Let $f\left(a^{*} a\right)>0$ and $a / \sqrt{f\left(a^{*} a\right)}=x$. Then $f\left(x^{*} x\right)=1$. Now $\beta_{f}(a) \geq\left|f\left(a^{2}\right)\right| /$ $\sqrt{M f\left(a^{*} a\right)}$. If $a$ is in $S(A)$, then we have $\sqrt{|f(a)|^{2} / M^{2}} \leq \sqrt{f\left(a^{2}\right) / M} \leq \beta_{f}(a)$; that is, $|f(a)| \leq M \beta_{f}(a)$. Hence, $|f(a)|^{2} \leq M f\left(a^{*} a\right) \leq M^{2} \beta_{f}(a)^{2}$ so that $|f(a)| \leq$ $M g(a)$. This proves that $f$ is $g$-bounded. Futhermore, for all $a$ in $A$ and for any positive linear functional $f$ on $A$,

$$
\sup _{a \in A}\left\{\frac{|f(a)|^{2}}{f\left(a^{*} a\right)}\right\} \leq|f|_{g} \leq M
$$

where $M$ is a positive constant (depending only on $f$ ). Therefore we may suppose that $M=\sup _{a \in A}\left\{|f(a)|^{2} / f\left(a^{*} a\right)\right\}$ so that we obtain $|f|_{g}=\sup _{a \in A}\left\{|f(a)|^{2} / f\left(a^{*} a\right)\right\}$.

Remark 2.2. If $A$ has an identity 1, then every positive linear functional $f$ is $g$ bounded. This follows directly by Theorem 2.1 and the Cauchy-Schwarz inequality. Further, if $A$ is unital with isometric involution, then by Lemma 2.1 it follows that $\|f\|=|f|_{g}=f(1)$.

We use the fact of Remark 2.2 and Theorem 2.1 to give a characterization of $g$-bounded linear functionals when the given algebra has approximate identity and isometric involution.

Theorem 2.2. If $A$ has isometric involution and approximate identity, a positive linear functional $f$ is $g$-bounded if and only if it is continuous.

Proof. If $f$ is $g$-bounded, then for all $a$ in $A,|f(a)| \leq|f|_{g} g(a) \leq|f|_{g}\|a\|$ and consequently $f$ is continuous. Suppose conversely that $f$ is continuous, then for all $a$ in $A,|f(a)|^{2} \leq\|f\| f\left(a^{*} a\right)$ by 2.1.5 in [1]. Hence by Theorem $2.1 f$ is $g$-bounded.

Corollary 2.1. $|f|_{g}=\|f\|$, for all $f$ as in Theorem 2.2. 
Proof. Since $f$ is $g$-bounded we have $|f|_{g} \leq\|f\|$. Also the involution on $A$ is isometric. Therefore $|f(a)| \leq|f|_{g} g(a) \leq|f|_{g}\|a\|$. This implies that $\|f\| \leq|f|_{g}$ and hence $|f|_{g}=\|f\|$.

\section{3. $g$-BOUNDED FUNCTIONALS IN TERMS OF REPRESENTABLE FUNCTIONALS}

In the following theorem we establish a relationship between $g$-bounded and representable functionals. Here it is shown that the representable functionals are the positive $g$-bounded linear functionals and these are precisely the functionals generated by cyclic *-representations of the algebra.

Theorem 3.1. A positive linear functional $f$ on $A$ is representable if and only if it is g-bounded.

Proof. Suppose that $f$ is representable. Then by the definition of representable functionals there exists *-representation $\pi$ of $A$ on $H$ and a vector $x$ in $H$ such that for all $a$ in $A,|f(a)| \leq|\pi(a)|$ and $\|x\|^{2} \leq g(a)\|x\|^{2}$. Thus $f$ is $g$-bounded.

Suppose conversely that $f$ is $g$-bounded. If the norm on $A^{+}$, the unitization of $A$, is given by $\|(a, \lambda)\|=\|a\|+|\lambda|$, for all $a$ in $A$ and $\lambda$ in $\mathbb{C}$, then $A$ is isometrically and ${ }^{*}$-isomorphically embedded in the unital Banach *-algebra $A^{+}$. Since $f$ is $g$ bounded it follows from Proposition 2.1(i) that for all $a$ in $A,|f(a)|^{2} \leq|f|_{g} f\left(a^{*} a\right)$.

Let $f^{+}$be defined on $A^{+}$by $f^{+}((a, \lambda))=f(a)+\lambda|f|_{g}$, where $(a, \lambda)$ is in $A^{+}$. Then $f^{+}$is a linear functional on $A^{+}$, which also extends $f$ on $A$ and

$$
\begin{aligned}
f^{+}\left((a, \lambda)^{*}(a, \lambda)\right) & =f^{+}\left(a^{*} a+\bar{\lambda} a+\lambda a^{*}, \lambda \bar{\lambda}\right) \\
& =f\left(a^{*} a\right)+\bar{\lambda} f(a)+\overline{\lambda f(a)}+|\lambda|^{2}|f|_{g} .
\end{aligned}
$$

Thus,

$$
\begin{aligned}
f^{+}\left((a, \lambda)^{*}(a, \lambda)\right) & \geq f\left(a^{*} a\right)-2|\lambda|\left(|f|_{g}^{f}\left(a^{*} a\right)\right)^{1 / 2}+|\lambda|^{2}|f|_{g} \\
& =\left(\left(f\left(a^{*} a\right)\right)^{1 / 2}-|\lambda||f|_{g}^{1 / 2}\right)^{2} \geq 0 .
\end{aligned}
$$

Hence $f^{+}$is a positive linear functional on $A^{+}$.

Let $\mathcal{L}_{f}=\left\{a\right.$ in $A^{+}: f^{+}(b a)=0$ for all $b$ in $\left.A^{+}\right\}$. Then on the quotient space $A^{+} / \mathcal{L}_{f}$ we define $\left(x_{a}, x_{b}\right)_{f}=f^{+}\left(b^{*} a\right), a$ in $x_{a}, b$ in $x_{b}$. If $H_{f}$ is the completion of $A^{+} / \mathcal{L}_{f}$, then

$$
\left\|x_{f}\right\|_{f}^{2}=\left(x_{f}, x_{f}\right)_{f}=f^{+}\left((0,1)^{*}(0,1)\right)=|f|_{g} .
$$

For each $a$ in $A^{+}$let $\pi(a)$ be defined by $\pi(a) x_{b}=x_{a b}, x_{b} \in A^{+} / \mathcal{L}_{f}$. Then $\pi(a)$ is a well-defined linear operator on $A^{+} / \mathcal{L}_{f}$ and for $a$ in $A^{+}$

$$
\left\|\pi(a) x_{b}\right\|_{f}^{2}=\left(x_{a b}, x_{a b}\right)_{f}=f^{+}\left(b^{*} a^{*} a b\right) .
$$

It is easy to see that $\pi(a)$ is a bounded linear operator on $A^{+} / \mathcal{L}_{f}$ and so it has a unique extension to a bounded linear operator $\pi^{+}(a)$ on $H_{f}$. Also the mapping $a \rightarrow \pi^{+}(a)$ is an algebra homomorphism from $A^{+}$into $B\left(H_{f}\right)$ and for all $a, b$ and $c$ in $A^{+}$,

$$
\left(\pi(a) x_{b}, x_{c}\right)_{f}=\left(x_{b}, \pi\left(a^{*}\right) x_{c}\right)_{f} .
$$

Hence for all $x, y$ in $H_{f},(\pi(a) x, y)_{f}=\left(y, \pi\left(a^{*}\right) y\right)_{f}$ so that for all $a$ in $A^{+}$, $\left(\pi^{+}(a)\right)^{*}=\pi^{+}\left(a^{*}\right)$. Thus $\pi^{+}$is a ${ }^{*}$-representation of $A^{+}$on $H_{f}$ and hence the restriction map $\pi_{f}=\left.\pi^{+}\right|_{A}$ is a ${ }^{*}$-representation of $A$ on $H_{f}$. Next, for all $a$ in $A$

$$
\left(\pi(a) x_{f}, x_{f}\right)_{f}=\left(x_{(a, 0)}, x_{(0,1)}\right)_{f}=f^{+}((a, 0))=f(a) .
$$


Thus $f$ is a positive linear functional represented by the pair $\left(\pi_{f}, x_{f}\right)$, and the proof is complete.

The following proposition shows that our definition of representability of positive linear functionals is equivalent to the definition given by Rickart ([5], 4.5.5). The proof uses the construction of the proof of Theorem 3.1.

Proposition 3.1. If $f$ is a g-bounded positive linear functional on $A$ then $f$ can be represented by a pair $(\pi, x)$, where $x$ is a cyclic vector for the ${ }^{*}$-representation $\pi$, and moreover $|f|_{g}=\left\|x_{f}\right\|_{f}^{2}$.

Proof. By the definition of cyclic vector and Theorem 3.1 it is obvious that $x_{f}$ is a cyclic vector for the ${ }^{*}$-representation $\pi^{+}$of $A^{+}$on $H_{f}$. We claim that $x_{f}$ is a cyclic vector for the ${ }^{*}$-representation $\pi_{f}$ of $A$.

Since $f$ is $g$-bounded, there exists a sequence $\left\{a_{k}\right\}$ of elements of $A$ with $g\left(a_{k}\right) \leq 1$ such that $|f|_{g}=\lim _{k \rightarrow \infty} f\left(a_{k}^{*} a_{k}\right)$. Let $b_{k}=a_{k}^{*} a_{k}$. Then $b_{k}$ is in $S(A)$ and $g\left(b_{k}\right) \leq 1$, and since $\left|f\left(b_{k}\right)\right|^{2} \leq|f|_{g} f\left(b_{k} b_{k}^{*}\right) \leq|f|_{g}^{2}$, it follows that $\lim _{k \rightarrow \infty} f\left(b_{k}^{*} b_{k}\right)=|f|_{g}$.

Consider $\left\|x_{b_{k}}-x_{f}\right\|_{f}$. Then $\left\|x_{b_{k}}-x_{f}\right\|_{f}^{2}=f\left(b_{k}^{*} b_{k}\right)-2 f\left(b_{k}\right)+|f|_{g} \rightarrow 0$ as $k \rightarrow \infty$. Hence for any $a$ in $A^{+}$we have $\left\|x_{a b_{k}}-x_{a}\right\|_{f}=\left\|\pi^{+}(a)\left(x_{b_{k}}-x_{f}\right)\right\|_{f} \rightarrow 0$ as $k \rightarrow \infty$. However, $a b_{k}$ is in $A$ and hence $\pi_{f}(A) x_{f}$ is dense in $A^{+} / \mathcal{L}_{f}$. It follows that it is also dense in $H_{f}$. Thus $x_{f}$ is a cyclic vector for $\pi_{f}$. The equality $|f|_{g}=\left\|x_{f}\right\|_{f}^{2}$ follows from the proof of Theorem 3.1.

\section{4.}

In this section we question why $g$-bounded functionals may be interesting and supply an answer to this question as well. During the course of this research, we observed that in the case of $B^{*}$-algebras, $g$-bounded functionals coincide with the original norm. A $g$-bounded functional is more general in defining the state space of $A$ since the set $D(g)$ of $g$-bounded functionals is a subspace of $A^{*}$, the dual space of $A$.

Proposition 2.1 holds for any $B^{*}$-semi-norm. A $g$-bounded functional can be represented by a pair of cyclic vectors for the ${ }^{*}$-representations.

Let $\pi$ be a *-representation of $A$ on the Hilbert space $H$ and let $x$ be in $H$. Then any positive linear functional $f$, which is represented by $(\pi, x)$, defines a $g$-bounded positive functional $f_{T}$ on $A$ with $f_{T} \leq f$, where $T$ is a self-adjoint operator on $H$ such that $T \pi(A)=\pi(A) T$ and $0 \leq T \leq I_{H}$ (see Lemma 1.1 in [4]). Lemma 1.1 in $[4]$ is an extension of a result by Dixmier ([1], 2.5.1).

Another application of $g$-bounded functionals to the representation theory can be seen in characterizing representable functionals which can be represented by a topologically irreducible representation.

A positive linear functional $f$ is a pure state of $A$ (see Definition 2.1, [4]) if it is non-zero and $g$-bounded and if any $g$-bounded positive linear functional dominated by $f$ is of the form $\beta f$ with $\beta$ in $[0,1]$. The following result, which has been submitted to another journal, gives an application of $g$-bounded functionals.

Theorem 4.1 ([4]). Let $(\pi, x)$ be a cyclic representation of a positive linear functional $f$. Then $\pi$ is topologically irreducible and non-zero if and only if $f$ is a pure state of $A$.

An intriguing development in the representation theory of $g$-bounded functionals is Theorem 3.1 in [4] which states that the extreme points of $D(g)$ are the zero functional and the pure states of $A$. 
In closing, we would like to express our appreciation to the referee for his or her valuable suggestions which improved the clarity of our presentation.

\section{REFERENCES}

1. J. Dixmier, Les $C^{*}$-algèbres et leurs représentations, Gauthier-Villars, Paris, 1964. MR 30:1404

2. E. G. Effros, A decomposition theory for representations of $C^{*}$-algebras, Trans. Amer. Math. Soc. 107 (1963), 83-106. MR 26:4202

3. J. M. G. Fell, The structure of algebras of operator fields, Acta Math. 106 (1961), 233-280. MR 29:1547

4. A. K. Gaur, A characterization of representable functionals on complex Banach *-algebras, submitted, 1995.

5. C. E. Rickart, General theory of Banach algebras, Van Nostrand, Princeton, NJ, 1960. MR 22:5903

Department of Mathematics, Duquesne University, Pittsburgh, Pennsylvania 15282

E-mail address: gaur@mathcs.duq.edu 